\title{
Níveis de atividade física e fatores associados entre professores de medicina
}

\author{
Physical activity levels and associated factors among medical teachers
}

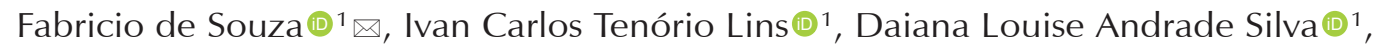
Samuel Eneias Pereira Viana ${ }^{1}$ 1, Francisca Maria Araújo da Silva ${ }^{1}$ 1, Betine Pinto Moehlecke Iser®1

${ }^{1}$ Universidade do Sul de Santa Catarina (Unisul), Tubarão, SC, Brasil.

RESUMO

Objetivo: Analisar os níveis de atividade física e os fatores associados entre professores do curso de medicina de uma universidade do Sul do Brasil.

Materiais e Métodos: Estudo epidemiológico transversal, realizado com professores do curso de medicina de uma universidade do Sul do Brasil. As variáveis sociodemográficas e de estado de saúde foram avaliadas por meio de entrevistas, a partir de um questionário adaptado do instrumento utilizado pela Vigilância de Fatores de Risco e Proteção para Doenças Crônicas por Inquérito Telefônico. Foram estimadas as razões de prevalência ajustadas de acordo com as características individuais da amostra, por Regressão de Poisson com variância robusta.

Resultados: A prevalência de docentes considerados fisicamente ativos no lazer foi de $44,2 \%$, fisicamente ativos no trabalho 40,4\%, fisicamente ativos em atividades domésticas 5,8\% e fisicamente ativos em atividades de deslocamento $2,6 \%$. A realização de atividades domésticas foi associada com a idade dos docentes, sendo maior entre os docentes com idade maior do que 45 anos (RP: 8,74 IC95\%: 1,03-74,31). Nenhuma outra característica sociodemográfica ou antropométrica foi associada à atividade física.

Conclusão: $O$ estudo concluiu que a maioria dos docentes do curso de medicina participantes deste estudo é considerada fisicamente ativa. Além disso, possuir idade superior a 45 anos demonstrou associação com maior quantidade de atividades domésticas.

Palavras-chave: atividade física; estilo de vida sedentário; inquéritos epidemiológicos; doenças crônicas não transmissíveis.

ABSTRACT

Objective: To analyze the levels of physical activity and the associated factors among teachers of a medical degree from an university in Southern Brazil.

Materials and Methods: A cross-sectional epidemiological study was conducted with teachers of a medical degree from an university in the South of Brazil. Sociodemographic and health status variables were evaluated by interviews, through a questionnaire adapted from the instrument used by the Risk and Protective Factors for Chronic Diseases by Telephone survey. Crude and adjusted prevalence rates according to the individual characteristics of the sample were estimated by Poisson regression with robust variance.

Results: The prevalence of teachers considered physically active on leisure time was $44.2 \%$, at work was $40.4 \%$, in domestic activities was $5.8 \%$ and in transportation was $2.6 \%$. The accomplishment of domestic activities was associated with the age of teachers, being higher among those aged more than 45 years (PR: 8.74 95\%Cl: 1.03-74.31). No other sociodemographic or anthropometric characteristics were associated with physical activity.

Conclusion: The study concluded that the majority of the medical degree teachers participating in this study were considered physically active. In addition, being more than 45 years old showed an association with greater amount of domestic activities.

Keywords: physical activity; sedentary lifestyle; health surveys; chronic non-communicable diseases. 


\section{INTRODUÇÃO}

A atividade física (AF) pode ser conceituada como qualquer movimento corporal produzido por músculos esqueléticos que exijam gasto de energia, incluindo atividades realizadas durante o trabalho, brincadeiras, realização de tarefas domésticas, viagens e envolvimento em atividades recreativas ${ }^{1}$. A Organização Mundial da Saúde (OMS) considera fisicamente ativo um adulto que faça pelo menos 150 minutos de AF de intensidade moderada, ou pelo menos 75 minutos de AF de intensidade vigorosa durante a semana, ou uma combinação equivalente de atividades de intensidade moderada e vigorosa ${ }^{1}$.

A OMS enfatiza a prática de AF como uma das principais alternativas para o combate a diversas doenças crônicas não transmissíveis, como doenças cardiovasculares, câncer e diabetes ${ }^{1,2}$. Os benefícios à saúde proporcionados pela prática regular de AF estão bem estabelecidos na literatura científica, incluindo melhor aptidão muscular e cardiorrespiratória, melhor saúde óssea e funcional, menor risco de desenvolvimento de doenças cardiovasculares, hipertensão arterial sistêmica, diabetes mellitus, depressão e diversos tipos de câncer ${ }^{1-3}$. Além disso, níveis adequados de AF promovem a diminuição dos efeitos fisiológicos naturais decorrentes do envelhecimento, aumentando a expectativa de vida ativa e limitando o desenvolvimento e a progressão de doenças crônicas e condições incapacitantes ${ }^{4}$. Destaca-se que maiores níveis de AF proporcionam maiores benefícios ${ }^{1}$. Por outro lado, menores níveis de AF estão associados com menor qualidade de vida ${ }^{5}$, dores no corpo ${ }^{6,7}$, indisposição e excesso de peso ${ }^{2,8}$.

Contudo, apesar dos benefícios proporcionados por níveis adequados de $\mathrm{AF}$, grande parcela da população mundial é considerada fisicamente inativa. A inatividade física é considerada um grave problema de saúde pública, visto que um em cada quatro adultos é considerado insuficientemente ativo no mundo (1,4 bilhão de pessoas) ${ }^{9}$. Globalmente, estima-se que a inatividade física gere um gasto de cerca de 54 bilhões de dólares em assistência médica direta, dos quais 57\% são incorridos pelo setor público e 14 bilhões adicionais são atribuíveis à perda de produtividade ${ }^{10}$.

Devido à gravidade do assunto, as ações que visam a diminuição dos níveis insuficientes de AF no mundo vem crescendo. A OMS desenvolveu o plano de ação global para a atividade física 2018-2030, o qual visa uma diminuição de $10 \%$ da AF insuficiente no mundo até 2025 e 15\% até $2030^{11}$. Esse plano possui orientações e enfatiza ações políticas efetivas e viáveis para aumentar a AF em todos os níveis. Nesse sentido, as pesquisas científicas possuem um papel fundamental, pois são encarregadas de produzir e compartilhar conhecimento ${ }^{11}$.
Sendo assim, identificar os níveis de AF e os fatores associados em diversas esferas da sociedade é imprescindível, pois possibilita definir alternativas para a implementação de ações apropriadas que estimulem a prática regular de AF, visando a diminuição dos custos de saúde ${ }^{10}$, a melhoria da qualidade de vida ${ }^{5}$ e da saúde das pessoas ${ }^{8}$. Os professores são considerados uma importante parcela da sociedade, principalmente os professores do ensino superior da área da saúde, visto que são influenciadores, responsáveis por desempenhar um importante papel na formação de futuros profissionais ${ }^{12}$. Apesar disso, estudos com essa população são escassos $^{8}$. Não foram encontrados estudos que avaliassem todos os domínios da AF exclusivamente em professores da área da saúde. Dessa forma, o objetivo deste estudo foi analisar os níveis de atividade física e os fatores associados entre professores do curso de medicina de uma universidade do Sul do Brasil.

\section{MATERIAIS E MÉTODOS}

Foi realizado estudo observacional transversal com docentes do curso de medicina de uma universidade do sul do Brasil. A amostra foi realizada por censo e incluiu todos os docentes que aceitaram participar do estudo. Os participantes que não completaram todas as etapas do estudo foram excluídos.

Este projeto foi aprovado pelo Comitê de Ética em Pesquisa da Universidade do Sul de Santa Catarina (Parecer número 516.469). O estudo foi conduzido de acordo com a Resolução 466/2012 do Conselho Nacional de Saúde e a declaração de Helsinki para estudos com seres humanos. Todos os participantes assinaram o termo de consentimento livre e esclarecido.

O estudo consistiu em uma entrevista e a identificação de medidas antropométricas. Uma equipe de profissionais e acadêmicos da área da saúde foi formada para coletar os dados. Todos os membros da equipe passaram por treinamento ministrado pelo pesquisador responsável. Uma administração prévia do questionário foi feita em população semelhante, em cerca de $10 \%$ da amostra estimada, para fins de calibração e padronização.

Um instrumento foi desenvolvido com base em questionários com perguntas validadas, além de perguntas feitas pelos autores para identificar as características dos participantes e seu estado clínico. O instrumento utilizado para avaliação da AF foi adaptado do questionário utilizado pela Vigilância de Fatores de Risco e Proteção para Doenças Crônicas por Inquérito Telefônico (VIGITEL) ${ }^{13}$, utilizando a recomendação da OMS para classificação no nível de AF¹.

Classificou-se como fisicamente ativo no tempo livre os participantes que praticaram pelo menos 150 minutos de AF 
de intensidade moderada por semana, ou pelo menos 75 minutos semanais de AF de intensidade vigorosa. A atividade com duração inferior a 10 minutos não foi considerada para efeito de cálculo da soma diária de minutos despendidos pelo indivíduo com exercícios físicos. Caminhada, caminhada em esteira, musculação, hidroginástica, ginástica em geral, natação, artes marciais e luta, ciclismo, voleibol/futevôlei e dança foram classificados como práticas de intensidade leve ou moderada; corrida, corrida em esteira, ginástica aeróbica, futebol/futsal, basquetebol e tênis foram classificados como práticas de intensidade vigorosa. Classificou-se como fisicamente ativo no deslocamento os participantes que praticaram AF no deslocamento equivalentes a pelo menos 150 minutos de atividade de intensidade moderada por semana. Classificou-se como fisicamente ativo no trabalho os participantes que realizaram esforços intensos na atividade de trabalho. Classificou-se como fisicamente ativo nas atividades domésticas os participantes que relataram serem responsáveis pela limpeza pesada do seu domicílio. Classificou-se como fisicamente inativos nos 4 domínios os participantes que não praticaram qualquer $\mathrm{AF}$ no tempo livre nos três meses anteriores à entrevista, que não realizaram esforços físicos intensos no trabalho, que não se deslocaram para o trabalho ou curso/escola caminhando ou de bicicleta, perfazendo um mínimo de 20 minutos no percurso de ida e volta, e que não foram responsáveis pela limpeza pesada de suas moradias. Classificou-se como possuindo hábito sedentário os participantes que despendem três ou mais horas diárias assistindo televisão ${ }^{13}$.

As medidas de peso e estatura foram obtidas por meio de questionamento direto com cada participante. A partir dessas medidas, o índice de massa corporal (IMC) foi obtido calculando-se a razão entre o peso e a estatura ao quadrado $\left(\mathrm{kg} / \mathrm{m}^{2}\right)$. Os pontos de corte para o IMC foram os propostos pela $\mathrm{OMS}^{2}$, que classifica como baixo peso os adultos que possuem o IMC abaixo de $18,5 \mathrm{~kg} / \mathrm{m}^{2}$, peso normal (eutróficos) os adultos que possuem o IMC entre $18,5 \mathrm{~kg} / \mathrm{m}^{2}$ e $24,9 \mathrm{~kg} / \mathrm{m}^{2}$, sobrepeso os adultos que possuem o IMC entre $25,0 \mathrm{~kg} / \mathrm{m}^{2}$ a $29,9 \mathrm{~kg} / \mathrm{m}^{2}$ e obesidade os adultos que possuem o IMC $\geq 30 \mathrm{~kg} / \mathrm{m}^{2}$. O excesso de peso, portanto, foi considerado para todos os participantes que possuíram o IMC $\geq 25 \mathrm{~kg} / \mathrm{m}^{2}$.

Os dados foram armazenados utilizando-se o programa estatístico EPI INFO ${ }^{\text {TM }}$ for Windows, versão 3.5.4 (Centers for Disease Control and Prevention, Atlanta, EUA), e analisados com o auxílio do programa STATA, versão 12 (Statacorp, Texas, EUA). As variáveis quantitativas foram descritas com medidas de tendência central (média) e dispersão (desvio padrão). As prevalências de inatividade física, prática de AF e outros desfechos de interesse foram apresentadas em proporção (\%) e intervalo de confiança de 95\%. Para os testes de associação foram utilizados os testes de Qui-quadrado para comparação de proporções e o teste t-Student para comparação de médias, ou equivalentes não paramétricos, com nível de significância de 5\%. A Razão de Prevalência (RP) foi estimada pela Razão de riscos, com IC 95\%. Razões de prevalência ajustadas foram estimadas pela Regressão de Poisson com variância robusta, com ajuste para idade, sexo e excesso de peso, tendo como categorias de referência: sexo feminino, menores de 45 anos, $I M C<25 \mathrm{~kg} / \mathrm{m}^{2}$.

\section{RESULTADOS}

A amostra inicial consistiu de 184 docentes que estavam contratados no momento em que se iniciou a coleta de dados. Destes, 14 foram excluídos por não completarem todas as etapas do estudo, dois se recusaram a participar da pesquisa e 12 não foram encontrados, resultando em uma amostra final de 156 docentes. Entre eles, 60,9\% eram do sexo masculino. A idade variou de 26 a 73 anos, com média de 45,2 $\pm 10,6$ anos. A maioria dos docentes era formado em medicina $(82,2 \%)$. Em relação à área de atuação profissional, a maioria atuava na área clínica $(70,1 \%)$, sendo que $11,7 \%$ eram cirurgiões. A maioria dos participantes deste estudo residiam na cidade de Tubarão (79,7\%). A Tabela 1 apresenta as características sociodemográficas da população em estudo.

Tabela 1. Variáveis sociodemográficas dos docentes do curso de medicina de uma universidade do sul do Brasil.

\begin{tabular}{llccc}
\hline Características & Categorias & $\begin{array}{c}\text { Total } \\
(\mathbf{n = 1 5 6})\end{array}$ & $\mathbf{\%}$ & IC 95\% \\
\hline \multirow{3}{*}{ Sexo } & Masculino & 95 & 60,9 & $52,8-68,6$ \\
& Feminino & 61 & 39,1 & $31,4-47,2$ \\
Profissária (anos) & $20-39$ & 57 & 36,7 & $29,0-44,6$ \\
& $40-59$ & 79 & 50,6 & $42,5-58,7$ \\
& $\geq 60$ anos & 20 & 12,8 & $8,0-19,1$ \\
& Médico & 125 & 82,2 & $75,2-88,0$ \\
& Enfermeiro & 8 & 5,3 & $2,3-10,1$ \\
& Dentista & 2 & 1,3 & $0,2-4,7$ \\
& Biólogo & 6 & 3,9 & $1,5-8,4$ \\
& Farmacêutico & 6 & 3,9 & $1,5-8,4$ \\
& Nutricionista & 2 & 1,3 & $0,2-4,7$ \\
& Bioquímicos & 2 & 1,3 & $0,2-4,7$ \\
& Fisioterapeuta & 1 & 0,7 & $0,0-3,6$ \\
& Fonoaudiólogo & 1 & 0,7 & $0,0-3,6$ \\
& Psicólogo & 1 & 0,7 & $0,0-3,6$ \\
& Pedagogo & 1 & 0,7 & $0,0-3,6$ \\
& Veterinário & 1 & 0,7 & $0,0-3,6$ \\
& Clínica & 108 & 70,1 & $62,2-77,2$ \\
& Cirúrgica & 18 & 11,7 & $7,1-17,8$ \\
& Pesquisa e ensino & 28 & 18,2 & $12,4-25,2$ \\
& Gestão de saúde & 1 & 1,3 & $0,2-4,6$ \\
& Patologista & 1 & 1,3 & $0,2-4,6$ \\
\hline
\end{tabular}

IC: Intervalo de confiança. 
Foi verificado IMC médio de $25,8 \pm 4,33 \mathrm{~kg} / \mathrm{m}^{2}$, o qual variou de $17,1 \mathrm{~kg} / \mathrm{m}^{2}$ a $42,6 \mathrm{~kg} / \mathrm{m}^{2}$, sendo que $1,9 \%$ foram considerados com baixo peso, $47,7 \%$ eutróficos, $35,5 \%$ com sobrepeso e 14,4\% obesos. Desta forma, 49,9\% dos docentes foram classificados com excesso de peso (IMC $\geq 25 \mathrm{~kg} / \mathrm{m}^{2}$ ), sendo $63,2 \%$ dos homens e $29,5 \%$ das mulheres, com diferença estatisticamente significativa entre os sexos $(p<0,001)$.

Em relação à prática de $\mathrm{AF}, 74,4 \%$ dos participantes do estudo afirmaram ter praticado algum tipo de exercício físico ou esporte nos três meses anteriores à entrevista. Os esportes mais praticados foram caminhada $(26,7 \%)$, musculação $(18,1 \%)$ e bicicleta (10,3\%). No que diz respeito à frequência e duração dessas atividades, 96,6\% praticaram a atividade pelo menos uma vez por semana, sendo que desses, $42 \%$ praticaram de 3 a 4 dias por semana e $42 \%$ praticaram de 1 a 2 dias na semana. No quesito tempo de duração da AF, $43,1 \%$ dos participantes relataram praticar a atividade por 60 minutos ou mais por dia.

Tabela 2. Classificação dos docentes do curso de medicina de uma universidade do sul do Brasil em relação aos domínios da atividade física.

\begin{tabular}{lccc}
\hline Domínios de atividade física & $\mathbf{n}$ & $\mathbf{\%}$ & IC $\mathbf{9 5} \%$ \\
\hline Ativos no lazer & 69 & 44,2 & $36,4-52,1$ \\
Ativos em atividades domésticas & 9 & 5,8 & $2,1-9,5$ \\
Ativos no deslocamento & 4 & 2,6 & $0,1-5,1$ \\
Ativos no trabalho & 63 & 40,4 & $32,6-48,2$ \\
Inativos & 4 & 2,6 & $0,1-5,1$ \\
Assistem TV $\geq 3$ h/dia & 2 & 1,3 & $-0,5-3,1$ \\
\hline
\end{tabular}

$\mathrm{n}=156$; IC: Intervalo de confiança.
Sobre a classificação do nível de AF, conforme os diferentes domínios, 44,2\% foram classificados como sendo ativos no lazer, $40,4 \%$ ativos no trabalho, 5,8\% ativos em atividades domésticas e 2,6\% ativos no deslocamento. Por outro lado, 2,6\% foram classificados como inativos e 1,3\% referiam assistir televisão 3 ou mais horas por dia (Tabela 2).

Foram realizadas múltiplas análises para identificação das prevalências e razões de prevalência brutas de cada domínio de AF, segundo as características individuais da amostra. Nessas análises, só foi observada diferença estatisticamente significativa entre os grupos na análise do domínio "atividades domésticas". A AF em atividades domésticas foi relatada por $5,3 \%$ dos professores do sexo masculino e por $6,6 \%$ dos professores do sexo feminino, sendo que a frequência foi cerca de oito vezes superior entre os professores de 45 anos ou mais de idade, em comparação aos que possuíam idade inferior a 45 anos (RP: 8,64; IC95\%: 1,11-67,45, $p=0,012$ ).

Em análise no modelo ajustado, segundo características específicas, verificou-se pequenas variações em relação aos dados brutos, sem mudanças significativas nas estimativas (Tabela 3). As principais mudanças na medida de associação foram observadas no domínio AF no lazer por sexo, ajustado por idade e excesso de peso, AF no deslocamento por idade, ajudado por sexo e excesso de peso, e AF no deslocamento por excesso de peso, ajustado por sexo e idade, com aumento na precisão das estimativas. A realização de AF no ambiente doméstico (realizar faxina no domicílio) manteve-se associada com a idade dos docentes $(p=0,047)$, mas com perda de precisão (aumento da amplitude do IC95\% 1,03-74,31).

Tabela 3. Prevalências e razões de prevalência ajustadas de cada domínio de atividade física segundo características individuais dos docentes do curso de medicina de uma universidade do sul do Brasil.

\begin{tabular}{|c|c|c|c|c|c|c|c|c|c|}
\hline \multirow[b]{2}{*}{ Variáveis } & \multicolumn{3}{|c|}{ Sexo } & \multicolumn{3}{|c|}{ Idade } & \multicolumn{3}{|c|}{ Excesso de peso } \\
\hline & $\begin{array}{c}\text { Masc } \\
\%\end{array}$ & $\begin{array}{c}\text { Fem } \\
\%\end{array}$ & RPa (IC 95\%) & $\begin{array}{c}<45 \\
\%\end{array}$ & $\begin{array}{c}\geq 45 \\
\%\end{array}$ & $\mathrm{RPb}(\mathrm{IC} 95 \%)$ & $\underset{\%}{\operatorname{Sim}}$ & $\begin{array}{c}\text { Não } \\
\%\end{array}$ & RPc (IC 95\%) \\
\hline Ativos no Lazer & 48,4 & 37,7 & $1,42(0,95-2,13)$ & 46,9 & 41,3 & $0,87(0,60-1,25)$ & 41,0 & 47,4 & $0,80(0,55-1,16)$ \\
\hline Ativos no Trabalho & 45,3 & 32,8 & $1,39(0,87-2,21)$ & 37,0 & 44,0 & $1,14(0,77-1,69)$ & 41,0 & 39,7 & $0,91(0,60-1,37)$ \\
\hline Ativos no Deslocamento & 4,2 & 0,00 & - & 1,2 & 4,00 & $2,55(0,31-21,39)$ & 2,6 & 2,6 & $0,53(0,08-3,31)$ \\
\hline Ativos em Atividades Domésticas & 5,3 & 6,6 & $0,51(0,13-1,99)$ & 1,2 & 10,7 & $8,74(1,03-74,31)^{*}$ & 7,7 & 3,8 & $1,65(0,33-8,15)$ \\
\hline Assistem TV $>3 \mathrm{~h}$ dia & 1,1 & 1,6 & $0,59(0,05-7,88)$ & 1,2 & 1,3 & $1,16(0,22-58,89)$ & 1,3 & 1,3 & $1,15(0,07-19,84)$ \\
\hline Inativos & 3,2 & 1,6 & $2,39(0,22-26,03)$ & 3,7 & 1,3 & $0,32(0,02-4,79)$ & 2,6 & 2,6 & $0,95(0,13-6,68)$ \\
\hline
\end{tabular}

RPa: Razão de prevalência ajustada por idade e excesso de peso; RPb: Razão de prevalência ajustada por sexo e excesso de peso; RPc: Razão de prevalência ajustada por sexo e idade. Regressão de Poisson com variância robusta.

IC: Intervalo de confiança.

$* p=0,047$ 


\section{DISCUSSÃO}

Este estudo verificou que $44,2 \%$ dos participantes da pesquisa foram considerados ativos no lazer, e 40,4\% ativos no trabalho, sem diferenças significativas por sexo e faixa etária. Apesar da baixa proporção de professores ativos nas atividades domésticas e no deslocamento (5,8 e 2,6\%, respectivamente), apenas $1 \%$ referiram hábito de assistir televisão por três ou mais horas por dia. Desta forma, a prevalência de inatividade física nos quatro domínios estudados foi baixa, de 2,6\%. Os profissionais com 45 anos ou mais de idade mostraram-se mais ativos fisicamente nas atividades domésticas. Metade da amostra estudada apresentou excesso de peso. Os resultados encontrados nesse estudo refletem o perfil da amostra estudada, sendo em geral profissionais de saúde, adultos de meia idade que, apesar da extensa carga horária de trabalho, são adeptos de um estilo de vida saudável, o que inclui a prática de atividade física no tempo livre, mesmo o tempo sendo escasso.

O nível de atividade física no lazer da amostra estudada se mostrou superior ao verificado na literatura. Segundo dados do inquérito VIGITEL, a prevalência de prática de AF entre adultos no lazer no Brasil é de $37 \%$, sendo maior entre homens $(43,4 \%)$ do que entre mulheres $(31,5 \%)$, e maior entre os mais novos, em ambos os sexos ${ }^{13}$. A diferença entre as proporções de ativos no lazer neste estudo em relação à prevalência nacional pode ser explicada pelas diferenças sociodemográficas das populações em estudo, visto que a prevalência de pessoas ativas no lazer é maior no sul do Brasil e em pessoas com maior nível de escolaridade ${ }^{13}$. Esse dado merece destaque, visto que todos os participantes deste estudo possuíam formação de nível superior, condizente com os resultados do inquérito VIGITEL.

No entanto, foi possível observar que a proporção de participantes desta pesquisa classificados como fisicamente ativos no lazer foi similar à população adulta residente na capital de Santa Catarina (43,6\%), mas com diferenças nas proporções entre homens e mulheres, visto que a proporção foi maior entre os homens $(50,7 \%)$ do que nas mulheres $(37,1 \%)^{13}$. Esse fato levanta a suspeita de que a localização geográfica possa ser o fator de maior influência no nível de AF. Porém, é importante destacar que a média de idade da população em estudo é relativamente alta (45 anos), o que pode ser um fator relevante, visto que o nível de AF diminui com o aumento da idade ${ }^{13}$. Esse destaque é corroborado por estudo que avaliou a AF de professores da rede pública de ensino de Londrina (PR), o qual identificou que apenas $28,1 \%$ dos docentes eram classificados como ativos no lazer, sendo verificado diferença significativa entre homens $(37,3 \%)$ e mulheres $(23,9)^{14}$. Esse resultado confronta a teoria de que a localização geográfica é o fator determinante no nível de $A F$, visto que ambos os estudos foram realizados no Sul no Brasil. Dessa forma, surge a hipótese de que essa diferença nas proporções possa ser explicada pelo fato de um grupo ser docente no nível superior e outro no ensino fundamental, ou ainda, pelo fato dos participantes desta pesquisa serem profissionais da área da saúde e possivelmente possuírem melhores condições financeiras ${ }^{13,14}$.

O alto nível de sedentarismo da população adulta é considerado um grave problema de saúde pública ${ }^{1,2}$, pois está associado a diversas doenças crônicas não transmissíveis, aumentando os riscos à saúde dessa população ${ }^{1,2}$, prejudicando a qualidade de vida ${ }^{5}$, comprometendo a renda familiar, além de onerar os sistemas de saúde pública e as iniciativas privadas ${ }^{10}$. A literatura científica que investiga os mais amplos fatores associados ao nível de AF da população adulta é vasta ${ }^{3,6,8}$. No entanto, não foram encontrados outros estudos que avaliassem os fatores associados à AF em todos os domínios entre docentes do ensino superior da área da saúde.

Analisando a prática de AF nos diferentes domínios, a única diferença estatisticamente significativa observada foi verificada no domínio das atividades domésticas, sendo que a proporção de docentes ativos neste domínio foi maior entre os participantes que possuíam idade igual ou superior a 45 anos. A diferença permaneceu após a realização de análise ajustada pelos possíveis fatores de confundimento (sexo e excesso de peso). Uma possível explicação para essa diferença pode ser atribuída a todas as mudanças que estão ocorrendo com o passar dos anos, as quais fazem com que cada vez menos se realize trabalhos domésticos, fato que era comum em todas as famílias há anos atrás ${ }^{15}$. Poucos estudos avaliaram a prática de atividades domésticas em populações adultas, mas alguns estudos que avaliaram encontraram diferenças em relação ao sexo, fato que não foi observado no presente estudo ${ }^{14,15}$, talvez por se tratar de amostra homogênea em relação à escolaridade e carga de trabalho.

Quando analisado a AF no trabalho, verificou-se que 40,4\% dos participantes deste estudo eram ativos, não sendo observado nenhuma diferença significativa em relação às análises realizadas. A proporção de participantes deste estudo classificados como ativos neste domínio foi superior a proporção de ativos verificada no estudo de Cunha e colaboradores $^{16}$, no qual $16,5 \%$ da população adulta de Goiânia era ativa no trabalho, com maior proporção de homens considerados ativos do que de mulheres (21,54 e $11,46 \%$, respectivamente $)^{16}$. A diferença observada entre os estudos talvez possa ser atribuída a diferenças nas características das populações estudadas, como o nível de escolaridade e a classificação econômica ${ }^{13}$. Entretanto, 
acredita-se que o principal fator que proporcionou essa diferença seja o fato de praticamente todos os participantes desde estudo serem professores e profissionais atuantes na área da saúde, o que lhes atribui uma jornada maior de trabalho devido a ter mais de uma atividade profissional ${ }^{17}$.

A proporção de ativos no deslocamento foi pequena, inferior à média nacional e à média da capital catarinense (13,4 e 14,5\%, respectivamente) ${ }^{13}$. A pequena proporção de ativos neste domínio talvez se deva ao fato dos participantes deste estudo terem características que estão associadas com menores níveis de AF no deslocamento, como dupla jornada de trabalho, alto nível de escolaridade, além de média de idade elevada ${ }^{13,15}$. É importante destacar que as várias formas de deslocamento de uma população dependem de vários fatores e não só por escolhas individuais, incluindo a acessibilidade, qualidade do transporte público e ambiente favorável para a mobilidade urbana ${ }^{18}$. Assim, outro possível fator para a baixa participação nas atividades de deslocamento pode ser atribuído ao aumento dos níveis de violência, que faz com que cada vez mais as pessoas procurem meios de transporte mais seguros para efetuar seus deslo camentos ${ }^{19}$.

Apenas 2,6\% da população em estudo foi classificada como sendo inativa nos quatro domínios. Este resultado é inferior à média nacional e da capital catarinense, ambos com $13,9 \%{ }^{13}$. A pequena frequência de inatividade física talvez possa ser explicada pelo fato de todos os participantes da pesquisa serem profissionais e docentes da área da saúde. Desse modo, é uma população que tem acesso as pesquisas científicas. Consequentemente, conhece os benefícios da prática regular da AF em relação à saúde e a qualidade de vida ${ }^{1-3,5}$. Devido a isso, todos os esforços no sentido de combater a inatividade física são válidos, dentre eles destacam-se as políticas públicas ${ }^{11}$, as mudanças nos hábitos diários $^{8,9}$ e o envolvimento em atividades comunitárias ${ }^{20}$ e familiares ${ }^{21}$.

Em relação aos hábitos marcadores de sedentarismo, como o tempo despendido assistindo à televisão, o resultado encontrado foi muito inferior à média nacional (24,6\%), e da capital catarinense $(25,2 \%)^{13}$. Essa diferença pode ser atribuída ao avanço tecnológico e às atribuições profissionais da população em estudo ${ }^{13,15}$. Entretanto, estes dados devem ser interpretados com cautela, podendo-se fazer duas considerações a respeito: a primeira é que este representa apenas uma parcela do tempo total de comportamento sedentário, sendo que o tempo sentado em frente ao computador ou outras telas, como tablet e celular ${ }^{13}$, até mesmo como atividade de trabalho, como no preparo de aulas, pode corresponder à grande parte da rotina desses profissionais, mas não detalhadas neste estudo. Por outro lado, há que se considerar que naturalmente a profissão médica e outras profissões de saúde, associadas à atividade de lecionar, requerem um engajamento do tempo diário, dessa forma não sobrando muito tempo para o hábito de assistir televisão.

Destaca-se no presente estudo a alta prevalência de docentes com excesso de peso (49,9\%), sendo que $63,2 \%$ dos docentes nessa classificação pertencia ao sexo masculino. Mesmo sendo alta, a prevalência geral de excesso de peso observada nesse estudo foi levemente inferior à média nacional $(54 \%)^{13}$. Entretanto, a proporção de homens do presente estudo classificados como possuindo excesso de peso foi um pouco superior à média nacional $(57,3 \%)^{13}$. Esse é um dado preocupante, pois além do excesso de peso ser um importante fator de risco para o desenvolvimento de muitas doenças ${ }^{22}$, também está associado à inatividade física ${ }^{8,23}$. A população em estudo pertence à área da saúde, consequentemente, espera-se que tenha conhecimento sobre todos os riscos para a saúde que o excesso de peso proporciona $^{22}$.

Como pontos fortes do presente estudo destaca-se a pequena quantidade de exclusões, que reduz a possibilidade de viés de seleção e contribui para a validade interna da pesquisa. A coleta de dados primários, por aplicação dos questionários em forma de entrevista, o que auxilia na interpretação dos resultados, minimiza os erros e permite ao estudo retratar a prevalência da prática de atividade física e sedentarismo entre os professores universitários. E por fim, o treinamento e a calibração da equipe de coleta de dados, assegurando padronização e qualidade na coleta.

Alguns limites na interpretação dos resultados desse estudo referem-se aos dados transversais, sendo que comportamentos relatados no momento da entrevista podem diferir de hábitos passados, que possam ter tido influência nas atitudes do presente. Assim, não se pode estabelecer relações temporais definidas entre os fatores que foram examinados e os principais desfechos. A precisão dos autorrelatos na averiguação dos hábitos diários e a auto avaliação das medidas de peso e estatura podem ser questionados, podendo incorrer em viés de informação. Entretanto, essas possíveis limitações podem ser consideradas minimizadas pelo fato dos participantes serem profissionais da área da saúde e terem conhecimento do assunto e um maior comprometimento com atividades científicas. A restrição da amostra apenas a docentes de uma única universidade, também poderia ser considerada uma limitação do estudo. No entanto, foi contemplada grande parte da população alvo do estudo, representando docentes da área da saúde com características diferentes, de modo que os dados podem ser válidos para outros docentes da área. 
Os resultados do presente estudo mostram que, em relação ao nível de atividade física dos professores do curso de medicina, este mostrou-se elevado em especial nos domínios de maior ingerência pessoal, como a realização de atividades físicas no lazer. Grande parcela dos docentes do curso de medicina praticaram algum tipo de exercício ou esporte nos três meses anteriores à entrevista, sendo que 96,6\% destes sujeitos praticaram pelo menos uma vez por semana, e a caminhada foi a atividade mais praticada. A população estudada também se mostrou fisicamente ativa no trabalho, demonstrando que os profissionais exercem atividades diversas de trabalho além da docência, sendo o tempo em sala de aula apenas uma parte da rotina dos professores. Entre os fatores associados à prática de atividade física, idade igual ou superior a 45 anos associou-se com maiores níveis de atividades domésticas. Por se tratar de um tema de extrema relevância, sugere-se a realização de mais estudos que investiguem os níveis de AF de docentes de diferentes áreas de atuação e regiões geográficas. Sugere-se também, que sejam realizados estudos experimentais que analisem os efeitos de intervenções nas condições de saúde e na qualidade de vida dessa população.

\section{AGRADECIMENTOS}

Agradecemos à CAPES (Coordenação de Aperfeiçoamento de Pessoal de Nível Superior) pela concessão de bolsa de estudos de Doutorado para o autor Fabricio de Souza.

\section{REFERÊNCIAS}

1. World Health Organization. Physical activity: Fact sheet; 2018 [Internet]. Geneva: WHO; 2018 [capturado em 6 mar. 2019]. Disponível em: https://www.who.int/en/news-room/fact-sheets/ detail/physical-activity.

2. World Health Organization. Global recommendations on physical activity for health; 2010. [Internet]. Geneva: WHO; 2018 [capturado em 6 mar. 2019]. Disponível em: http://apps.who.int/ iris/bitstream/10665/44399/1/9789241599979_eng.pdf.

3. Sallis JF, Bull F, Guthold R, Heath GW, Inoue S, Kelly P, Oyeyemi AL, Perez LG, Richards J, Hallal PC; Lancet Physical Activity Series 2 Executive Committee. Progress in physical activity over the Olympic quadrennium. Lancet. 2016;388(10051):1325-36. https://doi.org/10.1016/S0140-6736(16)30581-5

4. American College of Sports Medicine, Chodzko-Zajko WJ, Proctor DN, Fiatarone Singh MA, Minson CT, Nigg CR, Salem GJ, Skinner JS. American College of Sports Medicine position stand. Exercise and physical activity for older adults. Med Sci Sports Exerc. 2009;41(7):1510-30. https://doi.org/10.1249/ MSS.0b013e3181a0c95c

5. De Souza F, De Souza MMM, Schuelter-Trevisol F, Trevisol DJ. Relationships between physical activity, quality of life, and age in women attending social groups for the elderly. Sci Med. 2018;28(4):ID30301. https://doi.org/10.15448/19806108.2018.4.30301

6. Mohseni Bandpei MA, Ehsani F, Behtash H, Ghanipour M. Occupational low back pain in primary and high school teachers: prevalence and associated factors. J Manipulative Physiol Ther. 2014;37(9):702-8. https://doi.org/10.1016/j.jmpt. 2014.09.006

7. Palmlöf L, Holm LW, Alfredsson L, Magnusson C, Vingård E, Skillgate E. The impact of work related physical activity and leisure physical activity on the risk and prognosis of neck pain - a population based cohort study on workers. BMC Musculoskelet Disord. 2016;17:219. https://doi.org/10.1186/s12891-0161080-1

8. Silva CL, Souza MF, Rossi Filho S, Silva LF, Rigoni ACC. Atividade física de lazer e saúde: uma revisão sistemática. Mud Psico Saúde. 2017;25(1):57-65. https://doi.org/10.15603/2176-1019/mud. v25n1p57-65

9. Guthold R, Stevens GA, Riley LM, Bull FC. Worldwide trends in insufficient physical activity from 2001 to 2016: a pooled analysis of 358 population-based surveys with 1.9 million participants. Lancet Glob Health. 2018;6(10):e1077-e1086. https://doi. org/10.1016/S2214-109X(18)30357-7

10. World Health Organization.Physical activity: WHO launches Global Action Plan on Physical Activity. [Internet]. Geneva: WHO; 2018 [capturado em 6 mar. 2019]. Disponível em: https://www. who.int/news-room/detail/04-06-2018-who-launches-globalaction-plan-on-physical-activity.

11. World Health Organization. Noncommunicable diseases and their risk factors: Global action plan on physical activity 2018-2030: more active people for a healthier world. [internet]. Geneva: WHO; 2018 [capturado em 6 mar. 2019]. Disponível em: https:// www.who.int/ncds/prevention/physical-activity/global-actionplan-2018-2030/en/.

12. Weatherson KA, McKay R, Gainforth HL, Jung ME. Barriers and facilitators to the implementation of a school-based physical activity policy in Canada: application of the theoretical domains framework. BMC Public Health. 2017;17(1):835-51. https://doi. org/10.1186/s12889-017-4846-y

13. Ministério da Saúde (Brasil). Secretaria de Vigilância em Saúde. Departamento de Vigilância de Doenças e Agravos não Transmissíveis e Promoção da Saúde. VIGITEL Brasil 2017: vigilância de fatores de risco e proteção para doenças crônicas por inquérito telefônico. Brasília: Ministério da Saúde, 2019.

14. Dias DF, Loch MR, González AD, Andrade SM, Mesas AE. Insufficient free-time physical activity and occupational factors in Brazilian public school teachers. Rev Saude Publica. 2017;51: 68-78. https://doi.org/10.1590/s1518-8787.2017051006217

15. Villas-Boas S, Oliveira CS, Heras SL. Domestic tasks and gender: social representations of higher education students. Ex aequo. 2014;30:113-29. 
16. Cunha IC, Peixoto MRG, Jardim PCBV, Alexandre VP. Factors associated with physical activity in Goiania's adult population: surveillance through telephone interviews. Rev Bras Epidemiol. 2008;11(3):495-504. https://doi.org/10.1590/S1415$790 \times 2008000300016$

17. Chan L, McNaughton $H$, Weatherall M. Are physical activity levels of health care professionals consistent with activity guidelines? A prospective cohort study in New Zealand. JRSM Cardiovasc Dis. 2018;7-10. https://doi.org/10.1177/2048004017749015

18. Mielke GI, Hallal PC, Rodrigues GBA, Szwarcwald CL, Santos FV, Malta DC. Prática de atividade física e hábito de assistir à televisão entre adultos no Brasil: Pesquisa Nacional de Saúde 2013. Epidemiol Serv Saúde. 2015;24(2):277-86. https://doi. org/10.5123/S1679-49742015000200010

19. Powell-Wiley TM, Wong MS, Adu-Brimpong J, Brown ST, Hertenstein DL, Zenkov E, Ferguson MC, Thomas S, Sampson D, Ahuja C, Rivers J, Lee BY. Simulating the Impact of Crime on African American Women's Physical Activity and Obesity. Obesity (Silver Spring). 2017;25(12):2149-55. https://doi.org/10.1002/ oby. 22040

20. Anderson S, Currie CL, Copeland JL. Sedentary behavior among adults: The role of community belonging. Prev Med Rep. 2016;4:238-41. https://doi.org/10.1016/j.pmedr.2016. 06.014

21. Tebar WR, Oliveira CBS, Gil FCS, Saraiva BTC, Suetake VYB, Scarabottolo CC, Delfino LD, Fernandes RA, Christofaro DGD. Physical activity of parents and of their children: a systematic review of Brazilian sample studies - Report Card Brazil. Rev Bras Cinean Desemp Hum. 2018;20(4):532-42. https://doi. org/10.5007/1980-0037.2018v20n4p532

22. World Health Organization. Obesity and overweight [internet]. Geneva: WHO; 2018 [capturado em 16 mar. 2019]. Disponível em: https://www.who.int/news-room/fact-sheets/detail/obesityand-overweight.

23. Gray CL, Messer LC, Rappazzo KM, Jagai JS, Grabich SC, Lobdell DT. The association between physical inactivity and obesity is modified by five domains of environmental quality in U.S. adults: A cross-sectional study. PLoS One. 2018;13(8):e0203301. https:// doi.org/10.1371/journal.pone.0203301

\section{Autores:}

FABRICIO DE SOUZA

Educador Físico. Doutorando em Ciências da Saúde no Programa de

Pós-Graduação em Ciências da Saúde, Universidade do Sul de Santa Catarina (Unisul), Tubarão, SC, Brasil.

Orcid: http://orcid.org/0000-0001-5309-5515

E-mail: fabriciokarate@hotmail.com

IVAN CARLOS TENÓRIO LINS

Acadêmico do Curso de Medicina, Universidade do Sul de Santa Catarina (Unisul), Tubarão, SC, Brasil.

Orcid: http://orcid.org/0000-0002-1215-9508

E-mail: ivanlins82@hotmail.com

DAIANA LOUISE ANDRADE SILVA

Acadêmica do Curso de Medicina, Universidade do Sul de Santa Catarina (Unisul), Tubarão, SC, Brasil.

Orcid: http://orcid.org/0000-0001-5005-8989

E-mail: daianalouiseandradesilva@gmail.com
Samuel Eneias Pereira Viana

Acadêmico do Curso de Medicina, Universidade do Sul de Santa Catarina (Unisul), Tubarão, SC, Brasil.

Orcid: http://orcid.org/0000-0001-9314-3868

E-mail: samuelcuca22@gmail.com

Francisca MARIA ARAúJO DA SILVA

Acadêmica do Curso de Medicina, Universidade do Sul de Santa Catarina (Unisul), Tubarão, SC, Brasil.

Orcid: http://orcid.org/0000-0002-5327-0659

E-mail: fra.ma2008@hotmail.com

BetINE PINTO MOEHLECKE ISER

Cirurgiã-dentista. Doutora em Epidemiologia. Professora do Programa de Pós-Graduação em Ciências da Saúde, Universidade do Sul de Santa Catarina (Unisul), Tubarão, SC, Brasil.

Orcid: http://orcid.org/0000-0001-6061-2541

E-mail: betinee@gmail.com 